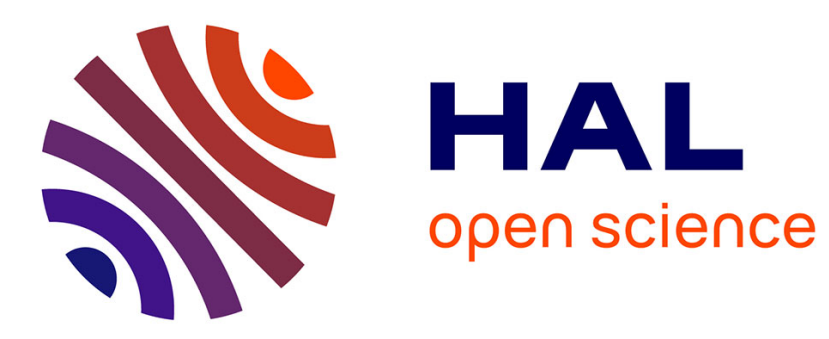

\title{
Broadcast-Multicast Single Frequency Network versus Unicast in Cellular Systems
}

Juan Vargas, Cédric Thienot, Christophe Burdinat, Xavier Lagrange

\section{To cite this version:}

Juan Vargas, Cédric Thienot, Christophe Burdinat, Xavier Lagrange. Broadcast-Multicast Single Frequency Network versus Unicast in Cellular Systems. 16th International Conference on Wireless and Mobile Computing, Networking and Communications, Oct 2020, Thessaloniki, Greece. 10.1109/WiMob50308.2020.9253389 . hal-03124600

\section{HAL Id: hal-03124600 https://hal.science/hal-03124600}

Submitted on 2 Feb 2021

HAL is a multi-disciplinary open access archive for the deposit and dissemination of scientific research documents, whether they are published or not. The documents may come from teaching and research institutions in France or abroad, or from public or private research centers.
L'archive ouverte pluridisciplinaire HAL, est destinée au dépôt et à la diffusion de documents scientifiques de niveau recherche, publiés ou non, émanant des établissements d'enseignement et de recherche français ou étrangers, des laboratoires publics ou privés. 


\title{
Broadcast-Multicast Single Frequency Network versus Unicast in Cellular Systems
}

\author{
Juan Vargas* $^{*}$, Cédric Thienot ${ }^{*}$, Christophe Burdinat *, Xavier Lagrange ${ }^{\dagger}$ \\ ${ }^{*}$ Enensys Technologies, Rennes, France \\ \{juan-carlos.vargas-rubio, cedric.thienot, christophe.burdinat $\} @$ enensys.com \\ ${ }^{+}$IMT Atlantique/IRISA, Rennes, France \\ xavier.lagrange@imt-atlantique.fr
}

\begin{abstract}
Video is an important factor of the load in cellular networks due to the growing popularity of streaming and linear services. In unicast transmission mode, the same data is transmitted as many times as the number of receivers demanding the same video content. Conversely, in broadcast transmissions using the Single Frequency Network (SFN) technique, a set of base stations perform synchronized transmission of the same waveform to a potentially infinite number of users. The objective of this study is to compare the performance of unicast and broadcast. More precisely, we determine the minimum number of users downloading the same data from which a broadcast transmission is more efficient than multiple unicast transmissions. In this paper, a model to calculate the Signal-to-Interference-plusNoise Ratio (SINR) in unicast and broadcast modes is presented, considering Poisson distributed base stations, path loss, fading, shadowing, trisectored antennas, SFN with a different number of base stations and beamforming in unicast mode. Results show that even when an SFN is formed by just 2 base stations and unicast transmissions are performed using beamforming with 8 antennas per sector, broadcast outperforms unicast when there are at least 8 users per cell demanding the same content.

Index Terms-Cellular Networks, SFN, Broadcast, Unicast, Beamforming, Trisectored antennas, video delivery.
\end{abstract}

\section{INTRODUCTION}

Video transmissions will account for 79 percent of the world's mobile data traffic in 2022 [1]. In order to provide a good quality of service, operators are searching for alternatives to reduce the radio resource utilization when several users are demanding the same video content on a given geographical area. Broadcast (BC) transmission using the Single Frequency Network (SFN) technique is an interesting solution to this problem. Using this technique, multiple Base Stations (BS) transmit identical waveforms at the same time to a potentially infinite number of users, thus reducing the interference. When a video is very popular, contents can be pushed via broadcast transmission and then stored in the cache of the receiver, even prior to user demand [2]. Thus, offloading the network and maintaining good service quality.

The Third Generation Partnership Project (3GPP) has standardized BC solutions since release 6 with the Multimedia Broadcast Multicast Service (MBMS) interface specification for 3G. It was conceived as a pre-planned and static mobile TV service based on SFN transmission [3]. In release 9, evolved MBMS (eMBMS) appeared as the LTE version of MBMS.
Release 10 introduced the eMBMS counting procedure. It allows the network to count the number of users interested in an MBMS service in a given SFN area [4, Chapter 11]. Release 12 Introduced eMBMS operation on demand (MooD) that allows automatic MBMS activation and deactivation. The option to perform BC transmission in only one cell was presented in release 13 as Single-Cell Point-to-Multipoint (SCPTM) [5, Chapter 19]. Release 14 introduced Further evolved MBMS (FeMBMS). It included a receive-only mode to allow devices without operator subscription to receive broadcast content. Other options as $100 \%$ carrier allocation and $200 \mu \mathrm{s}$ Cyclic Prefix (CP) were enabled [3]. In release 16, a further study item has evaluated the ability of FeMBMS to support an SFN of cells with a coverage radius of up to $100 \mathrm{~km}$ (implying even longer $\mathrm{CP}$ ) and mobile reception with speeds up to $250 \mathrm{~km} / \mathrm{h}$ [6]. $3 \mathrm{GPP}$ is considering in release 17 a mixed unicast/multicast mode to dynamically switch between both transmission modes [7].

In SFN, the network region in which BSs are synchronized to achieve an SFN transmission is called an SFN Area. It is generally a compact area without any hole, see Fig. 1. An SFN transmission is seen by a receiver as a single transmission suffering from multipath propagation. However, BC transmissions do not benefit from link adaptation. Therefore, they are aimed to cover the users with the worst channel conditions, i.e. users at the border of the SFN area. On the other hand, Unicast (UC) transmissions benefit from link adaptation and the possibility of using the beamforming technique but the same data is transmitted as many times as the number of users demanding the same service.

The problem is to identify at which point it becomes more efficient to transmit in BC mode rather than in UC mode from a resource utilization perspective. A user with a very low SINR can degrade the performance of BC transmissions. On the other hand, transmitting the same content several times in the same region is inefficient. The difficulty lies in the various irregularities in cellular systems, such as different BS densities, number of users demanding the same service, user's location, size of the SFN area, antennas capabilities, propagation effects, etc.

In [8], the authors propose a method to identify when it is convenient to change from $\mathrm{UC}$ to $\mathrm{BC}$ transmission based 
on the user with the lowest SINR. In [9], the authors prove the benefits of multicast over unicast when the average channel quality is good enough. However, both of these works consider BC transmission only in one cell. Models to calculate the SINR for a receiver in a SFN are presented in [10] and [11]. In [10] the model is based on a regular hexagonal lattice. On the other hand, authors in [11] consider Poisson distributed BS and use stochastic geometry to derive an approximation of the coverage probability in an infinite SFN network, but they consider omnidirectional antennas and do not consider shadowing.

In this study, a model to calculate the SINR for a receiver in UC and BC mode is developed. Additionally, a model to compare their efficiency in terms of resource utilization is provided. We use a Poisson Point Process (PPP) distribution for the BS location, instead of the traditional regular hexagonal lattice. This choice has been justified in [12] and [13]. We consider the characteristics of both transmission modes as well as path loss, fading, and log-normal shadowing. Utilizing Monte Carlo simulations we calculate the probability distribution of the SINR for each case. Then, we obtain an estimation on the number of users per BS demanding the same service from which the $\mathrm{BC}$ mode becomes more efficient that the UC mode, even when using beamforming.

The remainder of this paper is structured as follows. Section II presents the mathematical equations used to model the system, section III presents the derivation of the SINR for UC and BC transmission modes. In section IV we develop the model used to compare the efficiency of both transmission modes and the numerical results are analyzed in section $\mathrm{V}$. Finally, the conclusions are presented in section VI.

\section{SYSTEM MODEL}

We base our analysis on the reference model proposed by the 3GPP in [14]. The propagation model is based on Okumura-Hata-Cost231 with shadowing and fading. Threesector base stations are considered with adapted antennas. The 3 GPP model considers a limited number of base stations on a perfect hexagonal grid. To take into account the irregularity of operational networks, we consider BSs located following a PPP of density $\lambda$ as shown in Fig. 1. It is assumed that all BS use the same transmission power and carrier frequency.

\section{A. Noise Power}

The noise power $\left(P_{N}\right)$ is the aggregation of the thermal noise spectral density for the given occupied bandwidth $(W)$ and the receiver noise figure $(\gamma)$ and its value in $\mathrm{dB}$ is calculated as $P_{N d B}=\gamma+10 \log _{10}\left(k_{B} T_{k} W\right)$, where $k_{B}$ is the Boltzmann's constant and $T_{k}$ is the receiver system temperature.

\section{B. Path Loss}

The path loss in $\mathrm{dB}\left(P_{L d B}\right)$, valid for carrier frequencies between $1400 \mathrm{MHz}$ and $2600 \mathrm{MHz}$, is calculated as

$$
P_{L d B}=128.1+37.6 \log _{10}\left(r_{g}\right)+21 \log _{10}\left(\frac{f_{c}}{2}\right)
$$

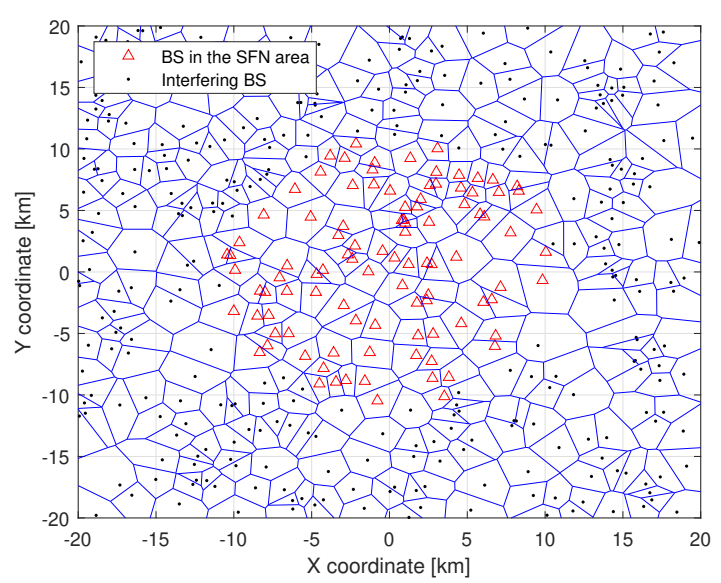

Fig. 1. SFN area of $100 \mathrm{BS}$ on a surface where BS are located following a PPP with density $\lambda=0.25 \mathrm{BS} / \mathrm{km}^{2}$.

where $f_{c}$ is the carrier frequency in $\mathrm{GHz}$ and $r_{g}$ is the receiver-BS geographical distance in $\mathrm{km}$. For a given frequency, (1) can be written as a function of the path loss exponent $\alpha$ and the path loss factor $k$ as $P_{L d B}=\alpha 10 \log _{10} r_{g}-10 \log _{10}(k)$, which in linear scale is $P_{L}=\frac{r_{g}^{\alpha}}{k}$.

\section{Fading and Shadowing}

Fading is modeled as an exponential random variable (r.v.) $h$ with unit rate. Shadowing is modeled as a log-normal r.v. $\exp (\chi)$, with $\chi$ following a normal distribution with zero mean and variance $\sigma^{2}$. Shadowing is usually characterized in terms of the standard deviation of its dB-spread $\sigma_{d B}=\frac{10}{\ln 10} \sigma$. Additionally, the r.v. $\chi$ is composed of a correlated part $\left(\chi_{c}\right)$, to account for the obstacles close to the receiver, and a non-correlated part $\left(\chi_{i}\right)$, corresponding to the obstacles independent for each BS, then, $\chi=\chi_{c}+\chi_{i}$ and $\sigma^{2}=\sigma_{c}^{2}+\sigma_{i}^{2}$. The correlation coefficient is defined as $\rho=\frac{\sigma_{c}^{2}}{\sigma^{2}}$.

\section{Shadowing effect on the BS density}

The received signal power $\left(P_{r x}\right)$ from a BS $i$ considering path loss, fading, shadowing and unit gain antennas, can be calculated as

$$
P_{r x}=P_{t x} k r_{g, i}^{-\alpha} \exp \left(\chi_{c}\right) \exp \left(\chi_{i}\right) h_{i},
$$

where $P_{t x}$ is the BS transmission power. By rewriting (2) as $P_{r x}=P_{t x} k\left(e^{-\frac{1}{\alpha} \chi_{i}} r_{g, i}\right)^{-\alpha} e^{\chi_{c}} h_{i}$, the uncorrelated shadowing can be considered as a random displacement of the BS original location changing the distance $r_{g, i}$ to $r_{i}=e^{-\frac{1}{\alpha} \chi_{i}} r_{g, i}$. Then, according to [15, Lemma 1], the new PPP defined by the transformed points $r_{i}$ is also a homogeneous PPP with density $\lambda^{\prime}=\lambda e^{2 \frac{\sigma_{i}^{2}}{\alpha^{2}}}$. In the remaining of this document, $r_{i}$ refers to the distance between the receiver and a BS $i$ considering the modified BS density $\lambda^{\prime}$, then

$$
P_{r x}=P_{t x} k r_{i}^{-\alpha} e^{\chi_{c}} h_{i} .
$$




\section{E. Trisectored Antennas}

Consider that each sector has a $120^{\circ}$ width. The antenna gain for each sector in the direction $\theta$, measured from the antenna boresight, can be calculated as stated in [14], as follows:

$$
G_{d B}(\theta)=G_{A}-\min \left\{12\left(\frac{\theta}{\theta_{3 \mathrm{~dB}}}\right)^{2}, G_{F B}\right\},
$$

where $G_{A}$ is the antenna gain in the boresight direction in $\mathrm{dB}, \theta_{3 \mathrm{~dB}}$ is the $3 \mathrm{~dB}$ beam width, $G_{F B}$ is the antenna front to back ratio and $-180^{\circ} \leq \theta \leq 180^{\circ}$. In this study it is assumed that the antenna boresight angles for the three sectors are $30^{\circ}$, $150^{\circ}$ and $270^{\circ}$ counterclockwise for all BSs.

\section{F. Beamforming}

The beamforming technique uses an array of antennas to increase the beam gain in a certain direction. This study considers BS with trisectored antennas in which each sector has a linear array of $M$ antennas capable of performing beamforming in unicast mode. The direction towards which the beam is pointed is characterized by the steering angle $\phi$ which is measured from the antenna boresight. The expression to calculate the array gain for one sector in the direction $\theta$ can be derived from $[16,17,18]$ as

$$
A(\theta, \phi)=\left\{\begin{array}{cc}
\frac{\sin ^{2}\left(M \frac{\pi}{2}(\sin (\phi)-\sin (\theta))\right)}{M \sin ^{2}\left(\frac{\pi}{2}(\sin (\phi)-\sin (\theta))\right)} G(\theta) & \theta \neq \phi \\
M G(\theta) & \theta=\phi
\end{array},\right.
$$

where $G(\theta)$ is given by (4) and $-60^{\circ} \leq \phi \leq 60^{\circ}$.

\section{G. SFN Model}

An SFN Area is composed of a number of BS $\left(N_{S F N}\right)$ that form a compact cluster and can perform synchronized transmission of the same waveform. In this study, we consider a large surface over which a certain number of BS $\left(N_{B S}\right)$ are located following a PPP of density $\lambda$. Two cases are studied. In the first one, we assume a very large SFN Area, e.g. countrywide deployment. Thus, all BS in the surface belong to the BC area $\left(N_{S F N}=N_{B S}\right)$ and the SINR perceived by a user placed in the origin of the plane is considered as a reference for all users, see [13, Remark 1.6.6]. In the second case, we consider a local SFN Area with a limited number of BS $\left(N_{S F N}<N_{B S}\right)$ defined as the $N_{S F N}$ BS closer to the origin of the plane. The BSs outside the BC area are considered as interferers. In this case, users are randomly placed in the SFN area, instead of the origin, to take into account the cases when the receiver is at the border of the $\mathrm{BC}$ area and perceive a smaller SINR.

\section{H. Interference in broadcast mode}

Consider a BS in an SFN area located at a distance $r_{i}$ from a receiver. Depending on the delay time with respect to the serving BS $\left(\tau_{i}\right)$ and the length of the Cyclic Prefix $\left(T_{C P}\right)$, the received signal power can be useful, partially useful or interference. Notice that we approximate the real distance $r_{g, i}$ to the modified distance $r_{i}$. It is assumed that the receiver is synchronized with the serving BS which is located at a distance $r_{s}$. Thus, $\tau_{i}=\frac{r_{i}-r_{s}}{c}$, where $c$ is the speed of light. Based on the model presented in [19], the usefulness of the signals received during an SFN transmission can be derived as

$$
\delta_{i}=\left\{\begin{array}{cc}
1 & 0 \leq r_{i}-r_{s} \leq c T_{C P} \\
\left(1+\frac{T_{C P}}{T_{u}}+\frac{r_{s}-r_{i}}{c T_{u}}\right)^{2} & c T_{C P}<r_{i}-r_{s} \leq c T_{f} \\
0 & r_{i}-r_{s}>c T_{f}
\end{array},\right.
$$

where $T_{u}$ is the useful symbol time and $T_{f}=T_{C P}+T_{u}$ is the total OFDM symbol time.

\section{SINR DERIVATION}

In this section, we derive the expressions of the SINR for a receiver in $\mathrm{BC}$ mode and $\mathrm{UC}$ mode based on the model presented in section II.

\section{A. SINR in unicast mode}

In UC mode, only the serving sector provides useful signal power $\left(P_{U C}\right)$. The other two sectors of the serving BS and all sectors from other base stations generate interference $\left(I_{U C}\right)$. Then, the SINR for a receiver in unicast mode can be calculated as

$$
S_{U C}=\frac{P_{U C}}{P_{N}+I_{U C}}=\frac{\hat{P}_{U C}}{\frac{P_{N}}{P_{t x} k e^{\chi_{c}}}+\hat{I}_{U C}},
$$

where $\hat{P}_{U C}$ and $\hat{I}_{U C}$ are the signal and interference power normalized by the factor $P_{t x} k e^{\chi_{c}}$ and are calculated as

$$
\hat{P}_{U C}=r_{s}^{-\alpha} h_{s} G\left(\theta_{s, t}\right),
$$

and

$\hat{I}_{U C}=r_{s}^{-\alpha} h_{s} \sum_{j=1 / j \neq t}^{3} G\left(\theta_{s, j}\right)+\sum_{i \epsilon \psi / i \neq s} r_{i}^{-\alpha} h_{i} \sum_{j=1}^{3} G\left(\theta_{i, j}\right)$,

where $\psi$ represents the PPP and the sub-indexes $s$ and $t$ represent the serving BS and the serving sector respectively.

\section{B. SINR in unicast mode considering beamforming}

It is assumed that the beam of the serving sector is steered in the direction of the receiver, then $\theta=\phi$. The SINR for a receiver in UC mode when considering beamforming can be calculated as

$$
S_{U C b}=\frac{P_{U C b}}{P_{N}+I_{U C b}}=\frac{\hat{P}_{U C b}}{\frac{P_{N}}{P_{t x} k e^{\chi_{c}}}+\hat{I}_{U C b}},
$$

where $\hat{P}_{U C b}$ and $\hat{I}_{U C b}$ are the signal and interference power normalized by the factor $P_{t x} k e^{\chi_{c}}$ and are calculated as

$$
\hat{P}_{U C b}=r_{s}^{-\alpha} h_{s} M G\left(\theta_{s, t}\right),
$$


and

$$
\begin{aligned}
\hat{I}_{U C b}=r_{s}^{-\alpha} & h_{s} \sum_{j=1 / j \neq t}^{3} A\left(\theta_{s, j}, \phi_{s, j}\right) \\
& +\sum_{i \epsilon \psi / i \neq s} r_{i}^{-\alpha} h_{i} \sum_{j=1}^{3} A\left(\theta_{i, j}, \phi_{i, j}\right),
\end{aligned}
$$

where the sub-indexes $s$ and $t$ represent the serving BS and the serving sector respectively.

\section{SINR in broadcast mode}

In BC SFN mode, all sectors of the BS belonging to the SFN area transmit the same waveform at the same time, and (6) is used to determine the usefulness of these signals. The SINR for a receiver in BC mode can be calculated as

$$
S_{B C}=\frac{P_{B C}}{P_{N}+I_{B C}}=\frac{\hat{P}_{B C}}{\frac{P_{N}}{P_{t x} k e^{\chi c}}+\hat{I}_{B C}},
$$

where $\hat{P}_{B C}$ and $\hat{I}_{B C}$ are the signal and interference power normalized by the factor $P_{t x} k e^{\chi_{c}}$ and are calculated as

$$
\hat{P}_{B C}=\sum_{i=1}^{N_{S F N}} \delta_{i} r_{i}^{-\alpha} h_{i} \sum_{j=1}^{3} G\left(\theta_{i, j}\right),
$$

and

$$
\begin{aligned}
\hat{I}_{B C}= & \sum_{i=1}^{N_{S F N}}\left(1-\delta_{i}\right) r_{i}^{-\alpha} h_{i} \sum_{j=1}^{3} G\left(\theta_{i, j}\right) \\
& +\sum_{i \epsilon \psi / i>N_{S F N}} r_{i}^{-\alpha} h_{i} \sum_{j=1}^{3} G\left(\theta_{i, j}\right) .
\end{aligned}
$$

Notice that in the case $N_{B S}=N_{S F N}$ the interference factor $\left(\hat{I}_{B C}\right)$ is reduced to the first term.

\section{RADio Resource Utilization}

\section{A. System Capacity and Outage Probability}

Consider a certain service with a capacity requirement $\left(C_{R}\right)$ in bits per second (bps). According to Shannon's theorem, the capacity of a system $(C)$ can be calculated as $C=W \log _{2}(1+S)$, where $W$ represents the system bandwidth and $S$ the SINR. Then, the number of resource blocks per time slot $\left(N_{R B}\right)$ needed to transmit a service with a capacity requirement $C_{R}$, can be calculated as

$$
N_{R B}=\frac{C_{R}}{W_{R B}} \frac{1}{\log _{2}(1+S)},
$$

where $W_{R B}$ is the bandwidth of a single resource block (RB). When the SINR at the receiver is very low, a lot of resources are needed to satisfy the capacity requirement. The network cannot allocate all resources to a single user. Thus, generally, users with a bad link quality are left out of service. The probability of a receiver being out of service is called the outage probability $p_{o}$ and is defined as $p_{o}=\mathbb{P}[S \leq s]$ which is the Cumulative Distribution Function (CDF) of the SINR. In this study, an outage probability threshold $\left(p_{o_{-} \max }\right)$ of $5 \%$ is allowed for both transmission modes.

\section{B. Resource utilization in unicast mode}

The Unicast mode uses link adaptation. Thus, to calculate the average number of RB per time slot used per UC user $\left(N_{R B_{U C}}\right)$, the expected value is estimated. Given that $p_{o_{-} \max }=\mathbb{P}\left[S_{U C} \leq s_{\min _{U C}}\right]=0.05$, then

$$
\begin{aligned}
N_{R B_{U C}} & =\mathbb{E}\left[\frac{C_{R}}{W_{R B}} \frac{1}{\log _{2}\left(1+S_{U C}\right)} \mid S_{U C} \geq s_{\min _{U C}}\right] \\
& =\frac{C_{R}}{W_{R B}} \int_{\text {smin }_{U C}}^{\infty} \frac{1}{\log _{2}(1+s)} f(s) d s \\
& =\frac{C_{R}}{W_{R B}} \Gamma_{U C},
\end{aligned}
$$

where $f(s)$ is the probability density function (PDF) of the SINR for a UC receiver and $\Gamma_{U C}$ represents the integral.

\section{Resource utilization in broadcast mode}

Broadcast transmissions do not benefit from link adaptation. Thus, transmissions are made aiming to cover the users with the worst channel conditions (low SINR). Since we allow a 5\% outage probability, the average number of RB used per BS per time slot in BC mode $\left(N_{R B_{B C}}\right)$ to transmit to a theoretically infinite number of $\mathrm{BC}$ receivers is given by

$$
N_{R B_{B C}}=\frac{C_{R}}{W_{R B}} \frac{1}{\log _{2}\left(1+s_{\min _{B C}}\right)}=\frac{C_{R}}{W_{R B}} \Gamma_{B C},
$$

such that $p_{o_{-} \max }=\mathbb{P}\left[S_{B C} \leq s_{\min _{B C}}\right]=0.05$ and $\Gamma_{B C}$ represents the second term, thus simplifying the expression.

\section{User Threshold}

Consider a certain number of users demanding the same service $\left(N_{U}\right)$. The total number of resource blocks needed for each transmission mode can be calculated as $N_{T_{R B U C}}=N_{U} N_{R B_{U C}}$ and $N_{T_{R B B C}}=N_{S F N} N_{R B_{B C}}$, for $\mathrm{UC}$ and $\mathrm{BC}$ respectively. We define the User threshold $\left(U_{T}\right)$ as the number of users per $\mathrm{BS}$ from which the $\mathrm{BC}$ mode consumes fewer resources than the UC mode $\left(N_{T_{R B B C}}<\right.$ $\left.N_{T_{R B U C}}\right)$. It can be derived as

$$
U_{T}=\frac{\Gamma_{B C}}{\Gamma_{U C}} .
$$

\section{NumericAl RESUlts}

The first objective of the simulations is to calculate the CDF of the SINR for all cases presented in previous sections and to do so, we use the Monte Carlo method. In each Iteration a new PPP for the BS location is generated and the SINR for a receiver in UC and BC modes are calculated based on equations presented in section III. To be compliant with 3GPP standards, most of the simulation parameters were taken from [14, Table C.6] and are given in Table I.

Fig. 2 shows the SINR CDF for a receiver in UC mode, including beamforming with a different number of antennas per sector $(M)$, and $\mathrm{BC}$ mode when considering a very large SFN area, i.e. $N_{B S}=N_{S F N}$ and a user located in the origin of the plane, for two different BS densities $\lambda_{1}=0.25 \mathrm{BS} / \mathrm{km}^{2}$ 
TABLE I

SIMULATION PARAMETERS

\begin{tabular}{||c|c||}
\hline \multicolumn{1}{|c|}{ Parameter } & Value \\
\hline \hline Area of the surface & $1600 \mathrm{~km}^{2}$ \\
\hline System Bandwidth $(W)$ & $5 \mathrm{MHz}$ \\
\hline Carrier Frequency $\left(f_{c}\right)$ & $2000 \mathrm{MHz}$ \\
\hline Receiver system temperature $\left(T_{k}\right)$ & $300 \mathrm{~K}$ \\
\hline UE Noise Figure $(\gamma)$ & $9 \mathrm{~dB}$ \\
\hline BS Transmission Power $\left(P_{t x}\right)$ & $20 \mathrm{~W}$ \\
\hline Useful OFDM Symbol Time $\left(T_{u}\right)$ & $66.7 \mu \mathrm{s}$ \\
\hline Cyclic Prefix Length $\left(T_{C P}\right)$ & $16.67 \mu \mathrm{s}$ \\
\hline Trisectored antenna gain $\left(G_{A}\right)$ & $15 \mathrm{dBi}$ \\
\hline Antenna frontback ratio $\left(G_{F B}\right)$ & $20 \mathrm{dBi}$ \\
\hline 3 dB beam width $\left(\theta_{3 d B}\right)$ & $655^{\circ}$ \\
\hline Shadowing Standard Deviation $\left(\sigma_{d B}\right)$ & $10 \mathrm{~dB}$ \\
\hline Shadowing Correlation Coefficient $(\rho)$ & 0.5 \\
\hline Noise Power $\left(P_{N}\right)$ & $-98 \mathrm{dBm}$ \\
\hline Path Loss Exponent $(\alpha)$ & 3.76 \\
\hline Path Loss Factor $(k)^{1}$ & 0.0295 \\
\hline 1 For distance in meters
\end{tabular}

1 For distance in meters.

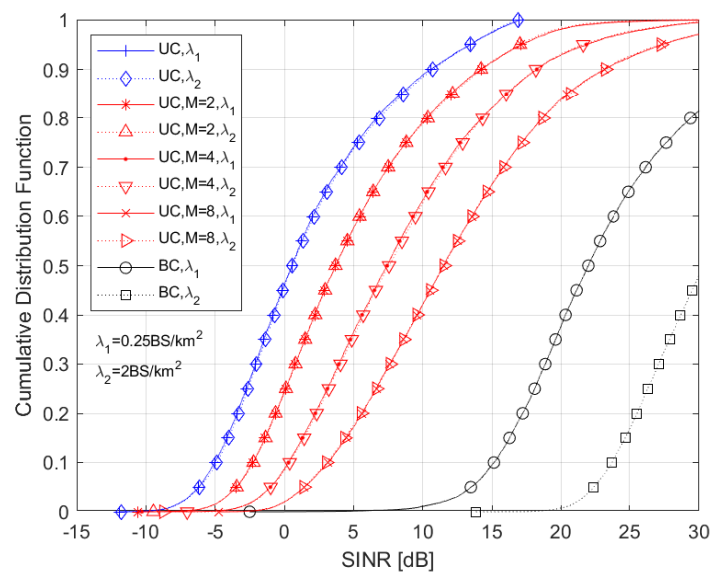

Fig. 2. Cumulative Distribution Function of the SINR for Unicast mode, including beamforming with different number of antennas $M$, and Broadcast mode, considering all BS as part of the SFN area, for different values of BS density $\lambda$.

and $\lambda_{2}=2 \mathrm{BS} / \mathrm{km}^{2}$. As expected, the beamforming technique increases the SINR in UC mode, the higher the number of antennas per sector $(M)$, the higher the SINR, thanks to the increased signal power, as seen in Fig. 3. However, the BC mode provides a higher SINR that all UC cases due to the reduced interference power as seen in Fig. 4. Notice in Fig. 2 that the BS density doesn't have a significant effect when transmitting in UC mode. As seen in Fig. 4 the interference power increases when increasing the BS density attenuating the effect of the increase in signal power. In contrast, when transmitting in $\mathrm{BC}$ mode, the higher the BS density the higher the SINR. A higher number of BS in the SFN area increases drastically the signal power while maintaining a low interference.

Fig. 5 shows the CDF of the SINR in BC mode when the SFN area is formed by the $N_{S F N}$ BSs closer to the origin of the plane. BSs outside the $\mathrm{BC}$ area generate interference. Two

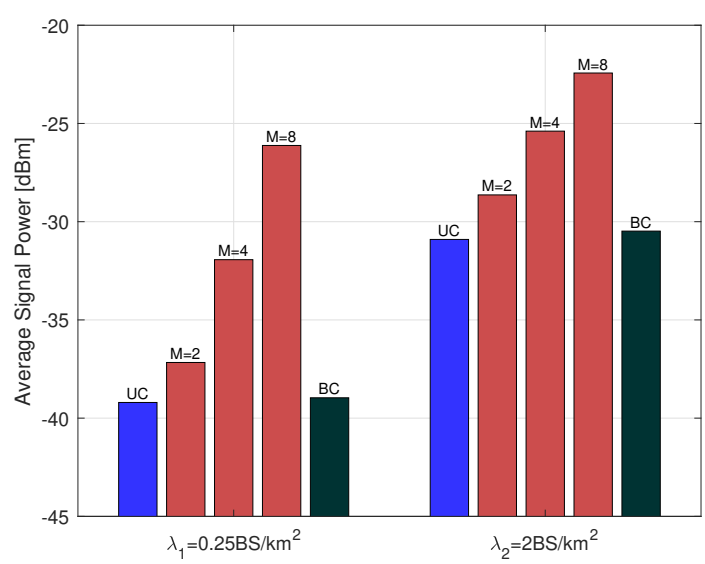

Fig. 3. Average Signal power for Unicast mode, including beamforming with different number of antennas $M$, and Broadcast mode, considering all BS as part of the SFN area, for different values of BS density $\lambda$.

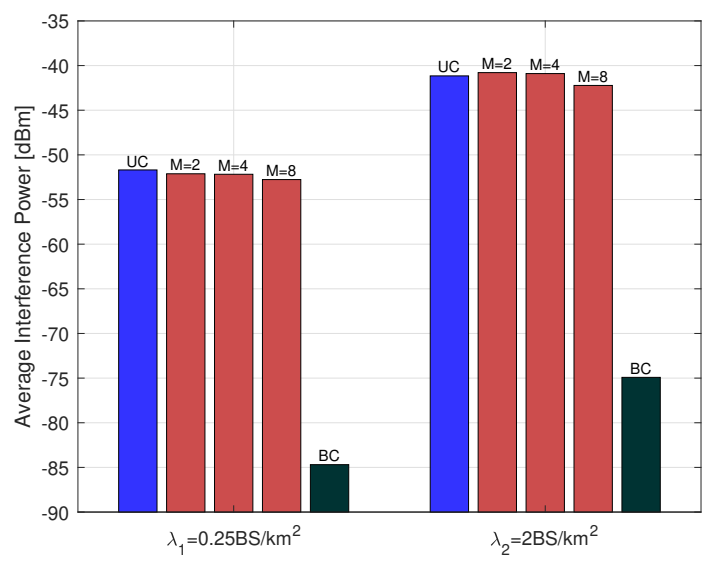

Fig. 4. Average Interference power for Unicast mode, including beamforming with different number of antennas $M$, and Broadcast mode, considering all BS as part of the SFN area, for different values of BS density $\lambda$.

different BS densities are considered $\lambda_{1}=0.25 \mathrm{BS} / \mathrm{km}^{2}$ and $\lambda_{2}=2 \mathrm{BS} / \mathrm{km}^{2}$. In this case, we limit the maximum value of $N_{S F N}$ based on the maximum geographical size of the SFN area. It is set to half the size of the total simulated surface, i.e. $800 \mathrm{~km}^{2}$. This is $N_{S F N}=200 \mathrm{BS}$ and $N_{S F N}=1600 \mathrm{BS}$ for $\lambda_{1}$ and $\lambda_{2}$ respectively. As expected, the SINR increases with the number of BSs in the SFN area. Notice that for the same value of $N_{S F N}$, the BS density doesn't have an important effect on the SINR. However, if $N_{S F N}$ is fixed, with a reduced BS density the SFN covers a larger geographical area. On the other hand, if the objective is to increase the SINR in a surface with a fixed size, i.e. $800 \mathrm{~km}^{2}$, the operator should deploy more BS as part of the SFN area, thus increase the BS density. As seen in Fig. 5, an SFN with $N_{S F N}=1600$ provides a higher SINR than an SFN with $N_{S F N}=200$.

Finally, in Fig. 6, the User threshold $\left(U_{T}\right)$ versus the number of BS in the SFN area $\left(N_{S F N}\right)$ for different UC configurations 


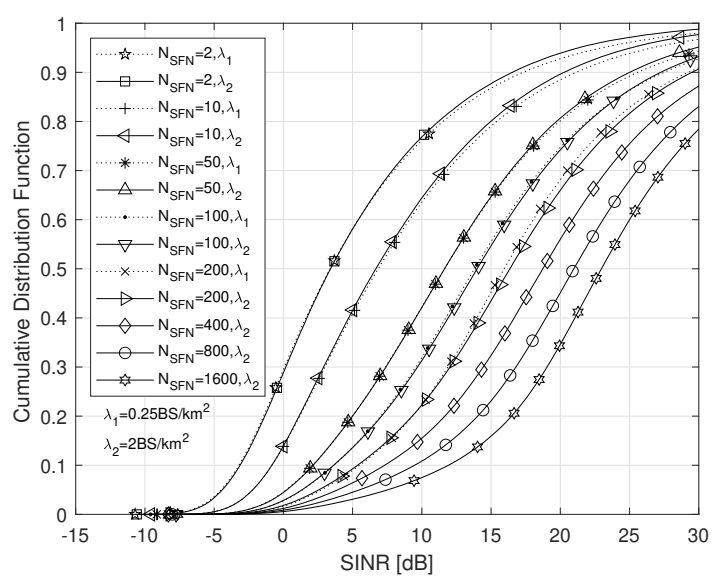

Fig. 5. Cumulative Distribution Function of the SINR for Broadcast mode considering SFN areas with a fixed number of BS $\left(N_{S F N}\right)$ for different values of BS density $\lambda$.

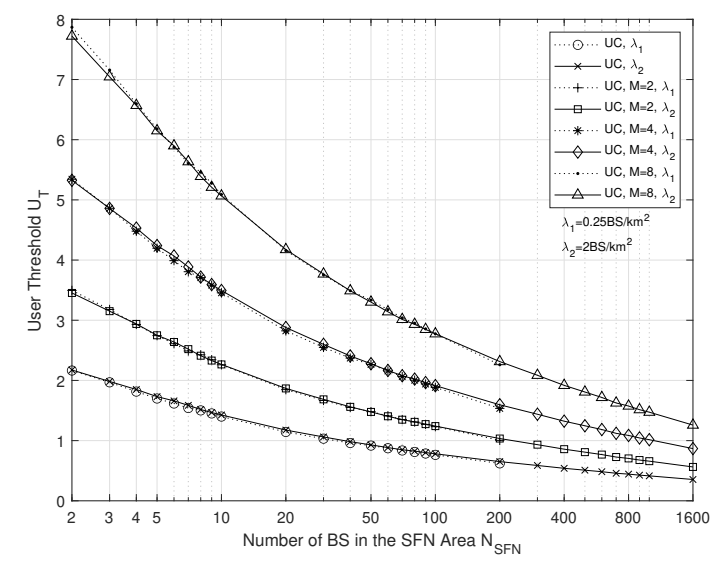

Fig. 6. User Threshold $\left(U_{T}\right)$ versus Number of BS in the SFN Area $\left(N_{S F N}\right)$ for different UC configurations and different values of BS density $\lambda$.

is presented. Notice that even when an SFN is formed by just 2 base stations and UC transmissions are performed using beamforming with 8 antennas per sector, BC outperforms UC when there are at least 8 users per cell demanding the same content. This information could be considered by the network in order to transmit using the most efficient transmission mode.

\section{CONCLUSION}

In this paper, we have provided a model to calculate the SINR for a receiver in a cellular network, formed by Poisson distributed BS with trisectored antennas, in Unicast mode and Broadcast SFN mode. Propagation effects as path loss, fading, and shadowing were considered. The study showed that when an SFN area covers a very large surface it outperforms UC transmission even when using beamforming with eight antennas per sector. It also showed that an increased BS density increases the SINR in SFN mode but doesn't have an important effect in UC mode. We analyzed the case when an SFN has a fixed number of BS. We provide an estimation of the number of users per BS demanding the same service from which the BC mode consumes fewer resources than the UC mode. It was found that the user threshold is below 8 users in all cases. Future work could consider sub-grouping techniques in SFN mode.

\section{REFERENCES}

[1] Cisco White Paper. Cisco Visual Networking Index: Global Mobile Data Traffic Forecast Update, 2017-2022. White Paper. ENST Paris, February 2019.

[2] Hao Feng, Zhiyong Chen, and Hui Liu. "Performance Analysis of Push-Based Converged Networks With Limited Storage". In: IEEE Transactions on Wireless Communications 15.12 (Dec. 2016), pp. $8154-8168$.

[3] David Gomez-Barquero et al. "Point-to-Multipoint Communication Enablers for the Fifth Generation of Wireless Systems". In: IEEE Communications Standards Magazine 2.1 (Mar. 2018), pp. 53-59.

[4] SeungJune Yi et al. Radio protocols for LTE and LTE-advanced. Wiley, 2012. $339 \mathrm{pp}$.

[5] Erik Dahlman, Stefan Parkvall, and Johan Sköld. 4G LTE-Advanced Pro and The Road to $5 G$ (Third Edition). Academic Press, Jan. 1, 2016, pp. 421-431.

[6] Jordi Joan Gimenez et al. " $5 \mathrm{G}$ New Radio for Terrestrial Broadcast: A Forward-Looking Approach for NR-MBMS". In: IEEE Transactions on Broadcasting 65.2 (June 2019), pp. 356-368.

[7] Mikko Saily et al. "5G Radio Access Networks: Enabling Efficient Point-to-Multipoint Transmissions". In: IEEE Vehicular Technology Magazine 14.4 (Dec. 2019), pp. 29-37.

[8] Seon Yeob Baek, Young-Jun Hong, and Dan Keun Sung. "Adaptive Transmission Scheme for Mixed Multicast and Unicast Traffic in Cellular Systems". In: IEEE Transactions on Vehicular Technology 58.6 (July 2009), pp. 2899-2908.

[9] Neila El Heni and Xavier Lagrange. "Multicast vs Multiple Unicast Scheduling in High-Speed Cellular Networks". In: VTC Spring 2008 - IEEE Vehicular Technology Conference. May 2008, pp. 2456-2460.

[10] Letian Rong, Olfa Ben Haddada, and Salah-Eddine Elayoubi. "Analytical Analysis of the Coverage of a MBSFN OFDMA Network". In: IEEE GLOBECOM 2008 - 2008 IEEE Global Telecommunications Conference. IEEE GLOBECOM 2008 - 2008 IEEE Global Telecommunications Conference. Nov. 2008, pp. 1-5.

[11] Ahmad Shokair et al. "Analysis of the Coverage Probability of Cellular Multicast Single Frequency Networks". In: 2019 International Conference on Wireless and Mobile Computing, Networking and Communications (WiMob). Oct. 2019, pp. 1-6.

[12] Bartłomiej Błaszczyszyn, Mohamed Kadhem Karray, and Holger Paul Keeler. "Using Poisson processes to model lattice cellular networks". In: 2013 Proceedings IEEE INFOCOM. Apr. 2013, pp. 773-781.

[13] François Baccelli. "Stochastic Geometry and Wireless Networks: Volume I Theory". In: Foundations and Trends in Networking 3.3 (2009), pp. 249-449.

[14] 3GPP. Evolved Universal Terrestrial Radio Access (E-UTRA); Radio Frequency $(R F)$ system scenarios. TR 36.942. Version 15.0.0. June 2018.

[15] Harpreet S. Dhillon and Jeffrey G. Andrews. "Downlink Rate Distribution in Heterogeneous Cellular Networks under Generalized Cell Selection". In: IEEE Wireless Communications Letters 3.1 (Feb. 2014), pp. 42-45.

[16] X. Lagrange. CIR cumulative distribution in a regular network. Research report. ENST Paris, 2000.

[17] Masood Maqbool, Marceau Coupechoux, and Philippe Godlewski. "Comparison of Various Frequency Reuse Patterns for WiMAX Networks with Adaptive Beamforming". In: VTC Spring 2008 - IEEE Vehicular Technology Conference. May 2008, pp. 2582-2586.

[18] T. T. Vu, L. Decreusefond, and P. Martins. "An analytical model for evaluating outage and handover probability of cellular wireless networks". In: The 15th International Symposium on Wireless Personal Multimedia Communications. Sept. 2012, pp. 643-647.

[19] A. Ligeti. "Coverage probability estimation in single frequency networks in presence of correlated useful and interfering components". In: Gateway to 21st Century Communications Village. VTC 1999-Fall. IEEE VTS 50th Vehicular Technology Conference (Cat. No.99CH36324). Vol. 4. Sept. 1999, 2408-2412 vol.4. 